\title{
АЛГОРИТМ АДАПТИВНОГО УПРАВЛЕНИЯ ОППОЗИТНЫМИ ИСПОЛНИТЕЛЬНЫМИ СИСТЕМАМИ С ПЕРЕМЕННОЙ СТРУКТУРОЙ
}

\section{ALGORITHM FOR ADAPTIVE CONTROL OF OPPOSED EXECUTIVE SYSTEMS WITH VARIABLE STRUCTURE}

Yu. Zhdanova

Summary. The article considers the specifics of solving control problems for special-design group-driven Executive systems used in anthropomorphic grippers. The necessary equipment of output links for building a closed control system is determined.

Keywords: anthropomorphic gripper, adaptive control, output links, group drive, closed system.

\author{
Жданова Юлия Ильдаровна \\ Старший преподаватель, МИРЭА - Российский \\ технологический университет \\ musrosjk@gmail.com
}

Аннотация. Рассмотрена специфика решения задач управления исполнительными системами с групповым приводом используемые в антропоморфных захватах. Определены необходимое оснащение выходных звеньев для построения замкнутой системы управления.

Ключевые слова: антропоморфный захват, адаптивное управление, выходные звенья, групповой привод, замкнутая система.

но NASA и General Motors для Robonaut 2 имеет пять параллельных ИС с 20 степенями подвижности [12]. Движение обеспечивается 16 двигателями, размещенными на звене манипулятора. Такое решение привело к увеличению его габаритов, и как следствие снижение его функциональных возможностей. Кроме того, необходимость обеспечения согласованного управления движением значительного числа выходных звеньев привело к резкому усложнению системы управления приводами.

Необходимость уменьшения размеров и массы приводов АЗ привело к формированию принципиально нового подхода к построению системы обеспечения движения звеньев ИС. Сформировался переход от индивидуального привода по каждой степени подвижности, к групповому приводу. Исключение кинематической зависимости движения звеньев, присущее групповому приводу, обеспечивается за счет технических решений основанных на: построении специальных систем передачи движения (СПД), реализации адаптивного управления движением выходными звеньями [13-15].

\section{Постановка задачи исслеАования}

Движение звеньев ИС с групповым приводом и адаптивным управлением кардинально отличается от реализуемых в системах с индивидуальным приводом. Один двигатель обеспечивает поочередным поворотом звеньев ИС, начиная с проксимального. При этом процесс взаимодействия ИС с ВО происходит изменение ее структурной схемы [16]. Каждое последующее звено ИС становится выходным при остановке предшествую- 
Таблица 1. Идентификация вариантов удержания ВО

\begin{tabular}{|c|c|c|c|c|c|}
\hline \multirow{2}{*}{$\begin{array}{l}\text { Вариант взаимодействия } \\
\text { (рис. 1) }\end{array}$} & \multicolumn{3}{|c|}{ параметров ИС } & \multirow{2}{*}{$\begin{array}{l}\text { Управляемый } \\
\text { параметр при } \\
\text { удержании }\end{array}$} & \multirow{2}{*}{ Объект управления } \\
\hline & $\overline{\varphi_{1}}$ & $\varphi_{2}$ & $h_{i}$ & & \\
\hline \multirow[t]{2}{*}{1} & 2 & 3 & 4 & 5 & 6 \\
\hline & + & 0 & $h_{2}$ & $R_{2}$ & 3вено 2 \\
\hline 6 & + & $\varphi_{2 K}$ & $h_{1}$ & $R_{1}$ & Звено 1 \\
\hline c & + & + & $h_{1}, h_{2}$ & $R_{2}$ & 3вено 2 \\
\hline д & $\varphi_{1 K}$ & + & $h_{2}$ & $R_{2}$ & 3вено 2 \\
\hline
\end{tabular}

A

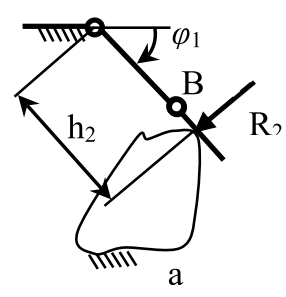

A $\varphi_{1}$

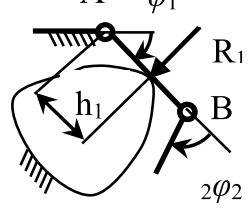

6
A $\varphi_{1}$

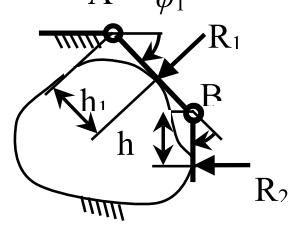

c

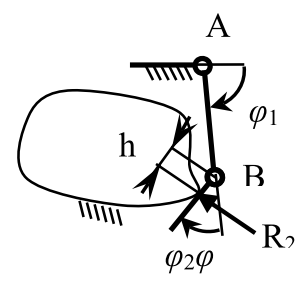

Д

Рис. 1. Варианты взаимодействия звеньев ИС на стадии удержания ВО

щего звена на ВО, или достижения им предельного угла относительного поворота $\varphi_{\mathrm{iK}}$.

В ИС с тремя звеньями последовательно реализуется три варианта структурных схем с одной степенью подвижности. В первом выходным является проксимальное звено, во втором - медиальное, в третьем - дистальное. В каждый момент времени объектом управления являются только одно звено.

Для ИС с двумя выходными звеньями возможно два варианта структурных схем. С движением только первого звена, а по достижению им поверхности ВО - движение второго. После успешной реализации фирмой Robotiq A3 с двумя оппозитными ИС, каждая из которых имеет два звена [17], интерес к такому варианту захвата со стороны потенциальных потребителей существенно вырос. Это в значительной мере обусловлено использованием АЗ в коллаборативных роботах [18], ориентированных на работу с ограниченной гаммой ВО. Реализованные системы управления обеспечивают захват и держание ВО имеющих симметричные формы и располагающиеся вдоль центральной оси АЗ. Управление захватом при взаимодействии с ВО недетерминированной поверхностью остается не решенной проблемой.

Процесс взаимодействия ИС с недетерминированным ВО разделяется на стадии: обхват, удержание [16]. Отдельно следует выделить стадию освобождения ВО. Специфика группового привода с адаптивным управлением определяет прерывание контакта звеньев в обрат- ной последовательности: от дистального к проксимальному и не требует специального алгоритма управления.

На стадии обхвата обеспечивается последовательное достижение всеми выходными звеньями ИС поверхности ВО. При этом силовое воздействие выходных звеньев на ВО незначительно и определяется сопротивлением движению в кинематических парах ИС. На стадии удержания относительное движение выходных звеньев в кинематических парах отсутствует. За счет двигателя создается контролируемое усилие со стороны выходных звеньев на ВО. Характер силового взаимодействия звеньев в пределах каждой ИС на стадии удержания зависит от используемой СПД.

\section{А^горитм управ^ения привоАами при взаимоАействии \\ с зафиксированным ВО}

Алгоритм управления приводами ИС зависит от наличия подвижности ВО. При отсутствии возможности его смещения под действием усилий со стороны выходных звеньев алгоритм управления приводом каждой из ИС является независимым. ИС имеет звенья 1 и 2, соединенные вращательной парой $B$ (рис.1). Звено 1 образует пару $A$ с основанием. Оси вращательных пар $A$ и $B$ параллельны. На стадии обхвата объектами управления являются звенья, последовательно начиная с проксимального, а управляемыми параметрами - углы относительного поворота звеньев $\varphi_{i}$. Управление осуществляется без обратной связи. На стадии удержания объект 


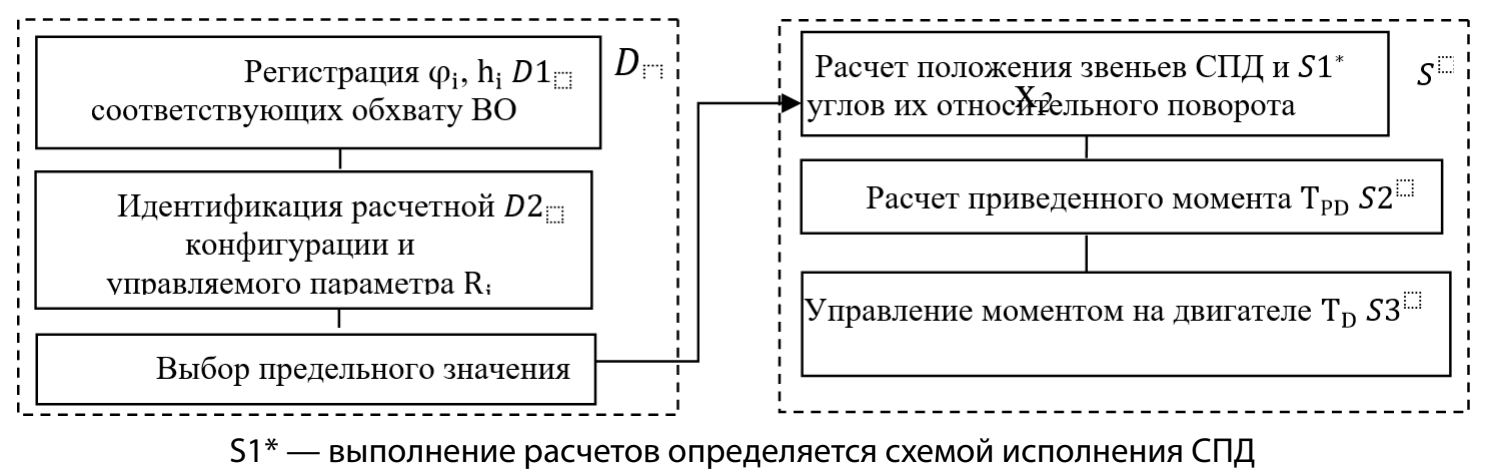

Рис. 2. Блок-схема управления выходным параметром $R_{i}$

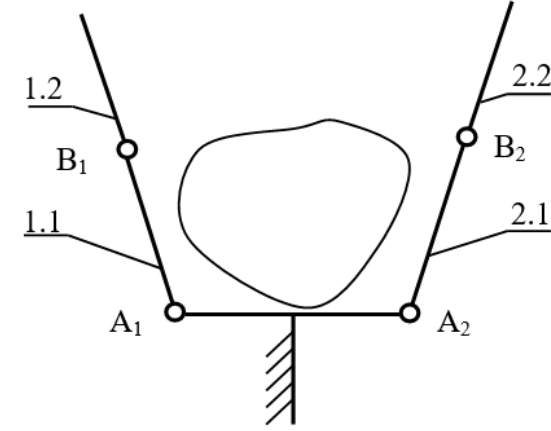

a

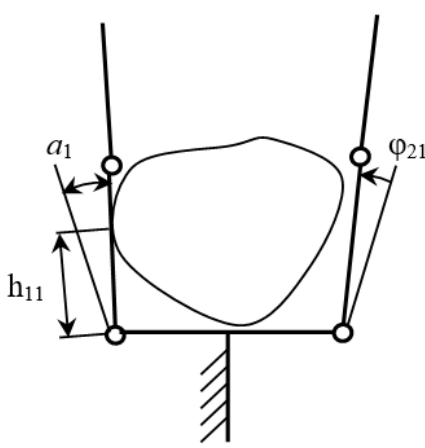

B

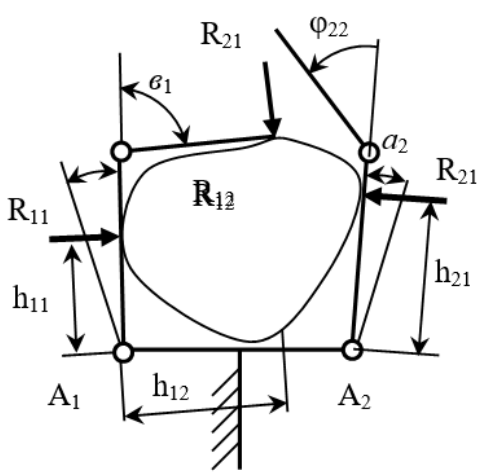

д

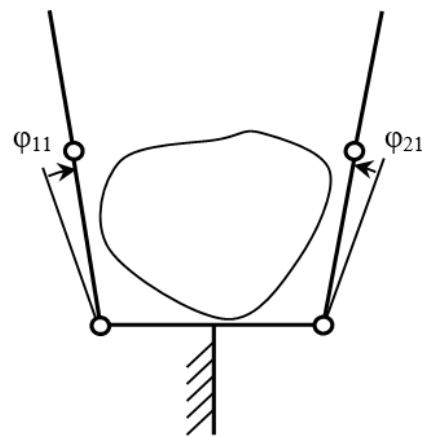

б

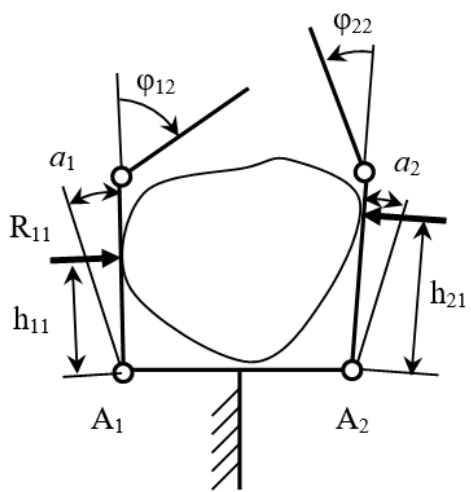

$\Gamma$

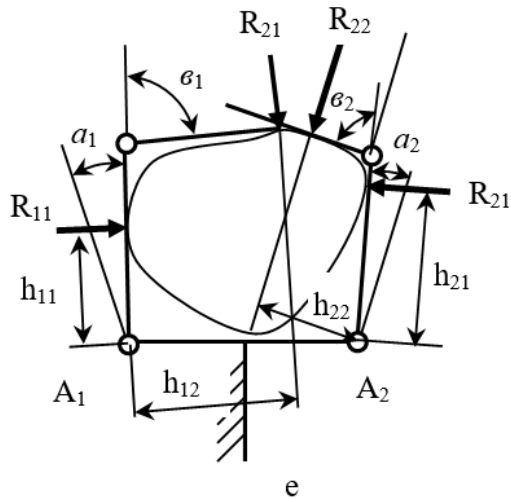

Рис. 3. Этапы обхвата звеньями ВО 
Таблица 2. Последовательность реализации обхвата и удержания ВО оппозитными ИС (рис. 3)

\begin{tabular}{|c|c|c|c|c|c|}
\hline \multirow{2}{*}{$\begin{array}{l}\text { Параметры } \\
\text { состояния }\end{array}$} & \multicolumn{5}{|c|}{ Этапы обхвата и удержания внешнего объекта } \\
\hline & I & II & III & IV & V \\
\hline Состояние звеньев & $\begin{array}{l}\varphi_{11} \neq 0, \varphi_{12}=0 \\
\varphi_{21} \neq 0, \varphi_{22}=0\end{array}$ & $\begin{array}{l}\varphi_{11}=a_{1}, \varphi_{12}=0 \\
\varphi_{21} \neq 0, \varphi_{22}=0\end{array}$ & $\begin{array}{l}\varphi_{11}=a_{1}, \varphi_{12} \neq 0 \\
\varphi_{21}=a_{2}, \varphi_{22}=0\end{array}$ & $\begin{array}{l}\varphi_{11}=a_{1}, \varphi_{12}=\mathrm{B} 1 \\
\varphi_{21}=a_{2}, \varphi_{22} \neq 0\end{array}$ & $\begin{array}{l}\varphi_{11}=a_{1}, \varphi_{12}=B_{1} \\
\varphi_{21}=a_{2}, \varphi_{22}=B_{2}\end{array}$ \\
\hline Объект управления & $\begin{array}{l}\text { Звено 1.1, } \\
\text { Звено 2.1 }\end{array}$ & Звено 2.1 & \begin{tabular}{|l} 
Звено 1.1, \\
Звено 1.1
\end{tabular} & Звено 1.2 & $\begin{array}{l}\text { Звено } 1.2 \\
\text { Звено } 1.2\end{array}$ \\
\hline $\begin{array}{l}\text { Момент на валу } \\
\text { двигателя }\end{array}$ & $\begin{array}{l}T_{P D 1.1}=k_{1}{ }^{*} T_{1.1} \\
T_{P D 21}=k_{2}{ }^{*} T_{21}\end{array}$ & $\begin{array}{l}T_{P D 1.11}=0 \\
T_{P D 2.1}=T_{P D 2} .1\end{array}$ & $\begin{array}{l}T_{P D 1.1}=f\left(R_{11}\right)=c \\
T_{P D 2 \|}=T_{P D 2 \|}\end{array}$ & $\begin{array}{l}T_{\text {PDI.1. }}=C_{1} \\
T_{\text {PD2 }}=d\end{array}$ & $\begin{array}{l}T_{\text {PDI.1V }}=e_{1} \\
T_{\text {PDZV }}=z\end{array}$ \\
\hline Управляемый параметр & $\begin{array}{l}\varphi_{11} \\
\varphi_{21}\end{array}$ & $\varphi_{21}$ & $\begin{array}{l}R_{11} \\
T_{p D 2 W}\end{array}$ & $\begin{array}{l}\mathrm{R}_{21} \\
\mathrm{~T}_{\mathrm{PD} 11}\end{array}$ & $\begin{array}{l}R_{12} \\
R_{22}\end{array}$ \\
\hline Схема взаимодействия & Pис. 3,6 & Рис.3, в & Pис.3, г & Рис.3, д & Pnc.3, e \\
\hline Процесс & \multicolumn{4}{|l|}{ Обхват } & Удержание \\
\hline
\end{tabular}

$k_{1}$ и $k_{2}-$ коэффициенты пропорциональности моментов $T_{i . I}$ и $T_{P D i . I}$

управления определяется вариантом взаимодействия выходных звеньев и ВО.

Всего возможно четыре варианта взаимодействия звеньев ИС с неподвижным недетерминированным ВО. Каждый вариант характеризуется исключительным сочетанием углов относительного поворота звеньев $\varphi_{i}$ и положением точки контакта $h_{i}$ (табл.1). Это позволяет идентифицировать вариант взаимодействия. В столбцах 1 и 2 символом «+» отображается наличие угла поворота, простановка $\varphi_{i K}$ соответствует достижение звеном предельного угла поворота определяемого конструкцией ИС.

Идентификация варианта удержания ВО позволяет определить:

- объект управления;

- условия, формирующие зависимость управляемого параметра от относительного положения звеньев ИС.

Процедура определения момента на двигателе выполняется в два этапа. На первом (часть $D$ блок - схемы рис. 2) фиксируются численные значения углов $\varphi_{i}$ определяющие конфигурацию ИС. Выделяется объект управления - звено, создающее усилие $R_{i}$ на поверхности ВО. Выбирается предельное значение $[R]$ исходя из задания на взаимодействие с ВО и рассчитывается моменты в кинематических парах. Действия, выполняемые на первом этапе, не зависят от используемого варианта построения СПД и определяются только параметрами ИС и положением ВО относительно АЗ.

На втором этапе (часть $S$ блок - схемы) выполняется расчет величины приведенного момента к валу двигателя $T_{P D}$ в функции зарегистрированных значений $\varphi_{i}, h_{i}$ управляемого параметра $R_{i}$ и конструктивных особенностей используемой СПД. Управление двигателем выполняются на основе сравнения значения $T_{P D}$ и текущего значения момента реализуемого на двигателе. В свою очередь зна- чение момента на двигателе сравнивается с допустимым $\left[T_{P D}\right]$, рассчитанным по величине предельно допустимого усилия воздействия на ВО $[R]$, и так же приведенного к двигателю. На этапе удержания управления осуществляется по замкнутой системе управления, обратная связь осуществляется опосредованно по величине $R_{i}$.

Для реализации алгоритма управления движением выходных звеньев необходимо оснащение вращательных пар энкодерами, а выходные звенья датчиками регистрации положения точек контакта.

\section{Алгоритм управления привоАами \\ при взаимоАействии \\ с не зафиксированным ВО}

Алгоритм управления приводами, при взаимодействии оппозитных ИС и ВО способным смещаться под действием звеньев, существенно отличается от предыдущего варианта. На рисунке 3 отображены этапы обхвата звеньями ИС поверхности ВО подвижного в плоскости параллельной изображению.

Первая ИС имеет выходные звенья 1.1 и 1.2 образующие вращательные пары $A_{1}$ и $B_{1}$. Углы относительного поворота в них соответственно $\varphi_{11}$ и $\varphi_{12}$ (рис. 3). Аналогично вторая, оппозитная ИС имеет звенья 2.1 и 2.2, пары $A_{2}$ и $B_{2}$, и углы относительного поворота $\varphi_{21}$ и $\varphi_{22}$.

Обхват ВО реализуется в четыре этапа. Для каждого характерным является оригинальное движение звеньев и величина необходимого момента. Исходное положение ВО относительно ИС представлено на рис. 3, а.

На первом этапе приводы обеспечивают одновременное изменение углов $\varphi_{21}$ и $\varphi_{22}$. Объектами управления являются звенья 1.1 и 2.1. Моменты создаваемые приводами в парах $A_{1}$ и $A_{2}$, соответственно $T_{1 . I}$ и $T_{2 . I}$, определяются сопротивлением движению в подвижных 
соединениях (табл. 2). На двигателях создаются моменты, соответственно $T_{P D 1 . I}$ и $T_{P D 2 . I}$. Зависимости между моментами на двигателях и моментами в кинематических парах $A_{i}$ линейные.

$$
T_{P D 1 . I}=k_{1} \times T_{1 . I}, T_{P D 2 . I}=k_{2} \times T_{2 . I}
$$

Это обусловлено отсутствуем относительного движения звеньев СПД. Управление параметрами $\varphi_{11}$ и $\varphi_{21}$ реализуется по разомкнутой системе. Этап завершается достижением любым из звеньев, 1.1 или 2.1, поверхности ВО. Например, звеном 1.1 (рис. 3, в). Положение точки контакта $-h_{11}$, фиксируется датчиком, установленным на рабочей поверхности звена, а угол поворота $\varphi_{11}=a_{1}$ энкодером. Привод первой ИС останавливается.

На втором этапе продолжается движение звена 2.1. Изменяется угол $\varphi_{21}$. Величина момента $T_{P D 2 . I I}$ сохраняется равной окончанию первого этапа $T_{P D 2 . I}$. Этап заканчивается при достижении звеном 2.1 поверхности ВО. Фиксируется положение точки контакта $h_{21}$ и угла поворота $\varphi_{21}=a_{2}$. Управления двигателем реализуется без обратной связи.

На третьем этапе (рис. 3, г) приводы ИС обеспечивают движение соответственно звеньев 1.2 и 2.2. При этом происходит относительное движение звеньев СПД в обоих ИС. Величина моментов, создаваемых на двигателях, определяется исходя из обеспечения неизменности положения ВО. Условие выполняется при равенстве моментов относительно соответственно точек $A_{1}$ и $A_{2}$ создаваемых действием звеньев 1.1 и 2.1 на ВО

$$
R_{11} \times h_{11}=R_{21} \times h_{21}
$$

Выполняется следующая процедура. Сравниваются значения моментов на приводах соответствующие окончанию первого этапа $T_{P D 1 . I}$ и $T_{P D 2 . I}$ При равенстве моментов выполнение условия (2) обеспечивается изначально. Движение звеньев реализуется при постоянных величинах моментов.

Если один из моментов превышает другой, например, при $T_{P D 2 . I}>T_{P D 1 . I}$ то рассчитывается усилие $R_{11}$ необходимое для соблюдения условия (2) по зависимости

$$
R_{11}=\frac{\left(T_{P D 2 . I} / k_{2}\right)}{h_{11}} .
$$

Наиболее конструктивным является контроль создаваемой величины $R_{l l}$ опосредовано, по величине момента приведенного к валу двигателя. В данном примере $T_{P D I . I I I}=f\left(R_{11}\right)$. Реализуется замкнутая система управления, при которой регистрируется момент на одном двигателе - $T_{P D 2 . I I I}$ а по его величине рассчитывается и регулируется момент на двигателе оппозитной ИС. При этом используется алгоритм, представленный в случае обхвата неподвижного ВО.

Управляемым параметром замкнутой системы на данном этапе является: момент $T_{P D 2 . I I I}$ и усилие $R_{11}$ контролируемое через момент $T_{P D 1 . I I I}$. Аналитические зависимости для расчета приведенного момента $T_{P D I . I I I}$ определяются конструкцией СПД.

Третий этап завершается достижением вторым звеном одной из ИС поверхности ВО. Например, звеном 1.2 (рис. 3, д), что регистрируется контактом в точке характеризуемой плечом $h_{12}$ относительно точки $A_{1}$. Зависимости между моментами, приведенными к валу двигателя, и в кинематических парах являются нелинейными.

На четвертом этапе движение в первой ИС отсутствует, при этом на двигателе создается момент соответствующий окончанию третьего этапа $T_{P D I . I I I}=c$. Привод второй ИС обеспечивает движение звена 2.2. При этом усилие реализуемое звеном 2.1 должно создавать момент в кинематической паре $A_{2}$ равный по величине тому, что создается силами $R_{11}$ и $R_{12}$ в паре $A_{1}$.

$$
R_{21} \times h_{21}=R_{11} \times h_{11}+R_{12} \times h_{12}
$$

Из последнего соотношения следует, что двигатель второй ИС должен обеспечить усилие $R_{21}$ равное

$$
R_{21}=\frac{\left(R_{11} \times h_{11}+R_{12} \times h_{12}\right)}{h_{21}}
$$

Знаки слагаемых в выражении (5) определяются по направлению момента создаваемого силами относительно точки $A_{1}$.

Далее выполняется процедура приведения силы $R_{21}$ к валу двигателя $T_{P D 2 . I V}=f\left(R_{21}\right)$.

Этап завершается при достижении звеном 2.2 ВО.

На четвертом этапе реализуется замкнутая система управления. Управляемым параметрами являются $T_{P D I}$. ${ }_{I V} и R_{21}$.

Если третий этап завершается более раним достижением звеном 2.2 ВО, то последовательность расчетов на четвертом этапе аналогична. При этом $T_{P D 2 . I V}=d$, а

$$
\begin{aligned}
& R_{12}=\frac{\left(R_{21} \times h_{21}+R_{22} \times h_{22}\right)}{h_{12}} \\
& \text { и } T_{P D 1 . I V}=f\left(R_{21}\right) .
\end{aligned}
$$

На этапе удержания (рис. 3, е) должно обеспечиваться уравновешивание моментов создаваемых действием звеньев оппозитных ИС на ВО 


$$
R_{11} \times h_{11}+R_{12} \times h_{12}=R_{21} \times h_{21}+R_{22} \times h_{22}
$$

Из соотношения (7) определяется величина $R_{22}$ обеспечивающая неизменное положение ВО

$$
R_{22}=\frac{\left(R_{11} \times h_{11}+R_{12} \times h_{12}-R_{21} \times h_{21}\right)}{h_{22}}
$$

Далее усилие $R_{22}$ приводится к моменту на валу двигателя $T_{P D 2 . V}=f\left(R_{22}\right)$. Момент на валу первой ИС сохраняется равным предшествующему этапу $T_{P D I . V}=T_{P D I . I V}$. Объектами управления являются звенья 1.2 и 2.2, управляемыми параметрами $R_{12}$ и $R_{22}$.

Особенность построения СПД с групповым приводом и адаптивным управлением является то, что при полном обхвате ВО объектом управления становится дистальное звено. В связи с этим при достижении звеньями 1.2 и 2.2 поверхности ВО силовое воздействие $R_{12}$ и $R_{22}$ определяемое величиной момента предшествующему возникновению контакта вторых звеньев ИС.

\section{Управления приво ами при по ъем ВО}

При подъеме ВО, для преодоления массовых сил, воздействие выходных звеньев на него должно быть увеличено. Следует принять $\max R_{i 2}=[R]$. В этом выражении левая часть есть большое из значений $R_{i 2}$ определенное на этапе удержания. На оппозитном выходном звене, имеющем меньшую величину $R_{i 2}$, ее зеачение определяется из соблюдения соотношения $R_{12} / R_{22}$ соответствующее этапу удержания.

\section{Зак^ючение}

Изложенный алгоритм адаптивного управления оппозитными ИС с групповым приводом является общим и не зависит от варианта построения СПД. В основе выполняемой последовательности действий используется принцип стабильности положения ВО. На этой основе выполняется определение усилий на выходных звеньях и соответствующих моментов в кинематических парах. Определение приведенных моментов к выходному валу привода основывается на специфике построения конкретной СПД и значениях углов относительного поворота звеньев зафиксированных на этапе обхвата. Для реализации изложенного алгоритма ИС должны быть оснащены энкодерами, фиксирующими углы относительного поворота звеньев, а выходные звенья датчиками регистрирующими положение точки контакта.

\section{Реферат}

Антропоморфные захваты с групповым приводом и адаптивным управлением обеспечивают взаимодействие с ВО при числе двигателей меньшем числа выходных звеньев. За счет переменности структуры ИС обеспечивается последовательное движение ее выходных звеньев от одного двигателя. Особенностью взаимодействия оппозитных ИС с ВО является перекрестная зависимость управления приводами. В качестве основы построения алгоритма управления двигателями при обхвате и удержании ВО принято отсутствии его смещения под действием выходных звеньев.

Выделены характерные этапы процесс обхвата и удержания ВО. В пределах каждого из них определены объекты управления и зависимости для расчета момента на приводе. Для обеспечения замкнутой системы управления необходима установка энкодеров в кинематические пары и датчиков для определения положения точки контакта на звеньях. Предложенный алгоритм позволяет формализовать процедуру формирования программы управления приводами оппозитных ИС с групповым приводом.

\section{ЛИТЕРАТУРА}

1. Сорокин В. Г. Вариант состава и структурной схемы базового блока автономного антропоморфного робота космического исполнения // Пилотируемые полеты в космос.— 2017. № 1 (22). С. 68-64.

2. Carbone G., González A. A numerical simulation of the grasp operation by LARM Hand IV: A three finger robotic hand. Robot. Comput. Integr. Manuf. 2011. 27. Pp.450-459.

3. Butterfaß J., Grebenstein M., Liu H., Hirzinger G. 2001. DLR-Hand II: Next Generation of a Dextrous Robot Hand. Proceedings of Intl Conf on Robotics and Automation (ICRA), Seoul; pp. 109-114.

4. Fukaya N., Toyama S., Asfour T., Dillmann R. Design of the TUAT/Karlsruhe humanoid hand.

5. Telegenov K., Tlegenov Y., Shintemirov A. An underactuated adaptive 3D printed robotic gripper. 2014 10th France-Japan/ 8th Europe-Asia Congress on Mecatronics (MECATRONICS2014-Tokyo), Tokyo, 2014, pp. 110-115. doi: 10.1109/MECATRONICS.2014.7018563

6. Mitsui K, Ozawa R, Kou T (2013) An under-actuated robotic hand for multiple grasps. In: International conference on robots and system (IR0S), Tokyo, pp 54755480 .

7. Chen X., Zhang W. COSA-ET finger: A coupled and self-adaptive underactuated robot finger with double springs and an empty-trip mechanism. $2016 \mathrm{IEEE}$ International Conference on Robotics and Biomimetics (ROBIO), Qingdao, 2016, pp. 539-543. doi: 10.1109/ROBI0.2016.7866378 
8. Ren Z., Zhou C., Xin S., Tsagarakis N., "HERI hand: A quasi dexterous and powerful hand with asymmetrical finger dimensions and under actuation," 2017 IEEE/RSJ International Conference on Intelligent Robots and Systems (IROS), Vancouver, BC, 2017, pp. 322-328

9. Badger J., Gooding D., Ensley K., Hambuchen K., and Thackston A. A Case study on Robonaut 2 ROS in Space The Complete Reference $1343-73$ Doi: https://doi. org/10.1007/978-3-319-26054-9

10. Yang Y., Zhang W., Xu X., Hu H., Hu J. (2017) LIPSA Hand: A Novel Underactuated Hand with Linearly Parallel and Self-adaptive Grasp. In: Zhang X., Wang N., Huang Y. (eds) Mechanism and Machine Science. ASIAN MMS2016, CCMMS2016. Lecture Notes in Electrical Engineering, vol 408. Springer, Singapore

11. Kang L., Seo J.-T., Kim S.-H., Kim W.-J., Yi B.-J. Design and Implementation of a Multi-Function Gripper for Grasping General 0bjects. Appl. Sci. 2019, 9, 5266. https: doi.org/10.3390/app9245266

12. Bridgwater L. B., Ihrke C. A., Diftler M. A., Abdallah M. E., Radford N. A., Rogers J. M., Yayathi S., Askew R. S., Linn D. M. The Robonaut 2 Hand — Designed To Do Work With Tools. NASA \& General Motors. 2012. pp. 1-2

13. Bandara D. S. V., Gopura R. A. R. C., Hemapala K. T. M. U., Kiguchi K. (2017). Development of a multi-DoF transhumeral robotic arm prosthesis. Medical Engineering and Physics, 48, 131-141. https://doi.org/10.1016/j.medengphy.2017.06.034

14. Kobayashi A., Yamaguchi K., Kinugawa J., Arai S., Hirata Y., Kosuge K. Analysis of precision grip force for uGRIPP (underactuated gripper for power and precision grasp), 2017 IEEE/RSJ International Conference on Intelligent Robots and Systems (IROS), Vancouver, BC, 2017. pp. 1937-1942.

15. Адаптивный привод группы звеньев захвата: пат. 185794 Рос. Федерация № 2018100908/09; заявл. 10.01.18; опубл. 19.12.18, Бюл. № 35. 5 с.

16. Bogdanov A, Permyakov A and Zhdanova Yu (2018) Synthesis of structural scheme of drive of adaptive multiple-link gripper MATEC Web of Conf. 161 03009. https:// doi.org: 10.1051/matecconf/201816103009

17. Choi M.-S., Lee D.-H., Park H., Kim Y.-J., Jang G.-R., Shin Y.-D., Park J.-H., Baeg M.-H., Bae J.-H. Development of multi-purpose universal gripper // Proc. of the 56th Annual Conf. of the Society of Instrument and Control Engineers of Japan (SICE). 2017. https://doi.org: 10.23919/SICE.2017.8105739.

18. Mathiassen K, Fjellin JE, Glette K, Hol PK and Elle 0J (2016) An Ultrasound Robotic System Using the Commercial Robot UR5. Front. Robot. Al3:1. https: doi: 10.3389/ frobt.2016.00001

( ) Жданова Юлия Ильдаровна ( musrosjk@gmail.com ).

Журнал «Современная наука: актуальные проблемы теории и практики»

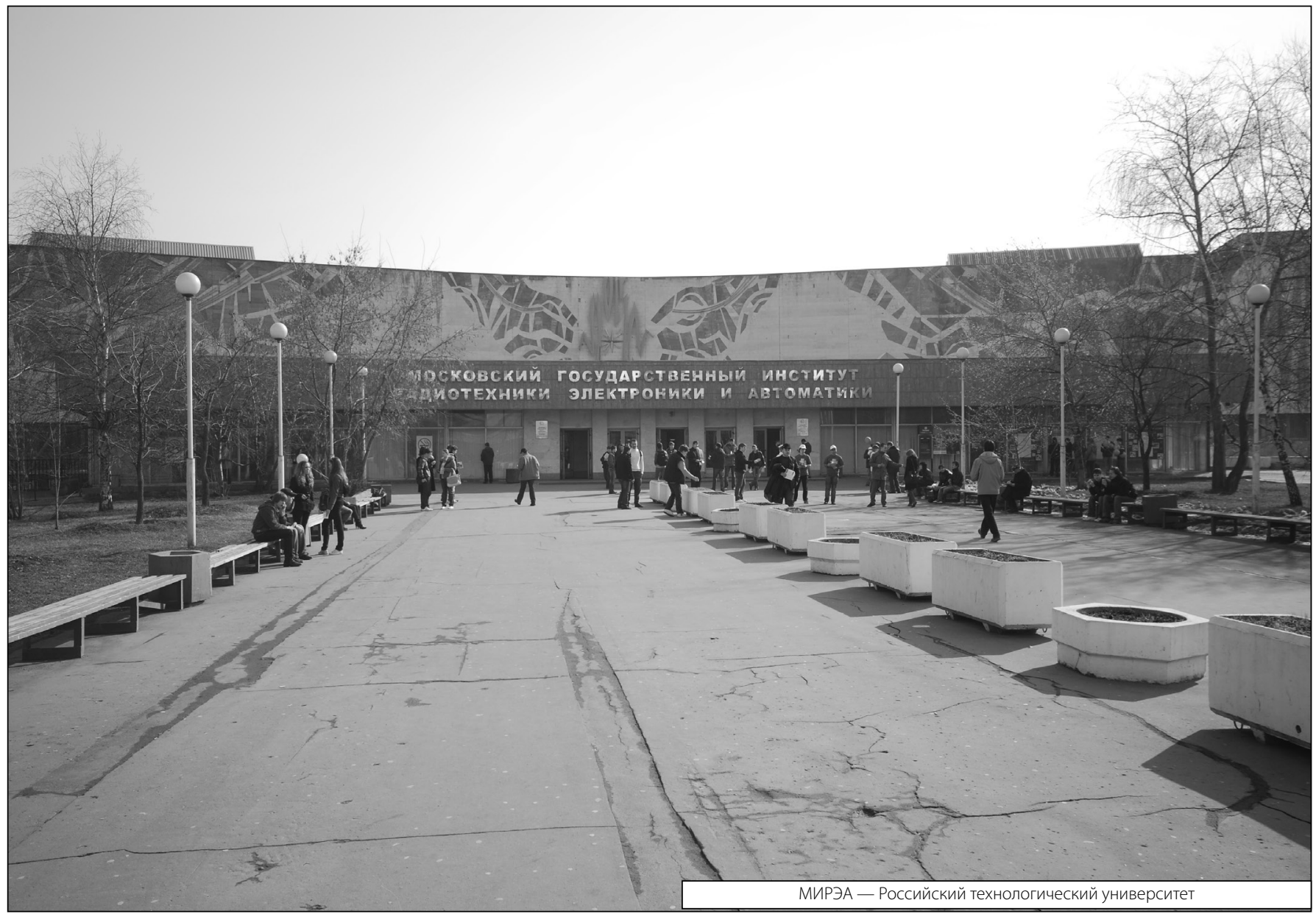

